\title{
Control of industrial crystallizers
}

\author{
Johan Jager, Herman J. M. Kramer, Esso J. de Jong \\ Laboratory for Process Equipment,
}

Sjoerd de Wolf, Okko H. Bosgra

Laboratory for Measurement and Control,

Arthur Boxman, Henk G. Merkus and Brian Scarlett

Department of Chemical Engineering, Delft University of Technology, Mekelweg 2, 2628 CD Delft (The Netherlands)

\begin{abstract}
The particle size distribution produced in particulate processes generally has a large impact on process economics, and the crystal size distribution produced in industrial crystallizers is no exception. Crystal Size Distribution (CSD) control is therefore desirable. Its establishment is the main objective of a joint research program between three research groups within the Delft University of Technology and several industrial participants. This paper presents a review of this project and intends (1) to illustrate the development of an on-line crystal size measuring technique, (2) to illustrate how information on CSD can be used to derive a dynamic process model and (3) to emphasize the need for effective process inputs and to make suitable suggestions in that direction.
\end{abstract}

\section{Introduction}

Crystallization from solution is an important unit operation in chemical engineering practice, giving a product of high purity at low energy costs. The process is employed in the production of bulk chemicals such as ammonium sulfate, sodium chloride and sucrose, and the production of fine chemicals such as aspartame. Usually the process yields a solid product which has a wide crystal size distribution (CSD). This CSD dictates the behaviour of the product in succeeding operations, such as filtration, drying, transport and storage, and is also defined by customer specifications. It is, therefore, desirable to control the CSD produced. This fact provides a motive to improve the state-of-the-art in CSDcontrol. Within a joint research programme between Delft University of Technology and several industrial participants [1], the continuous evaporative crystallization of ammonium sulfate in a draft-tube-baffled crystallizer serves as an example of the process.

The number of studies reporting the attempted development and experimental verification of CSD-control schemes is limited. This is due to the lack of a reliable on-line CSD measuring technique, which is the first requirement for process control, in a field where sieve analysis has long been applied as the major CSDmeasurement technique. Only comparatively recently have on-line CSD-measurement techniques, using laser diffraction, become commercially available. This technique is, however, restricted to slurries which contain solids at low concentration. It was applied, in a noteworthy attempt to establish CSD-control, by Randolph and coworkers [2] to measure the CSD in a side stream of a crystallizer containing fines at low concentration. A similar attempt was made by Rohani and Lee [3], who measured the fines concentration rather than the fines size distribution. Although the fines stream has some influence on the final product quality, it is obvious that, using this information only, the CSD cannot be fully controlled.

The second requirement lacking for CSD-control is closely related to the deficiency of on-line measuring techniques. Dynamic modelling of crystallization processes is still rudimentary, cspecially in predicting the effect of phenomena affecting the population in transient conditions such as crystal birth, crystal growth or other crystal number-affecting events. Consequently, previous CSD-control attempts were limited to single input-single output (SISO) CSD-controllers $[2,3]$, which were rather empirically designed. The main drawback of these simple proportional controllers is that transients and offsets of CSD-parameters, such as the mean and variance of the mass distribution, are still possible. In order to improve the control performance more inputs and outputs must be introduced which, in turn, requires more powerful multivariable controllers to account for interactions between them. Most design methods for 
multivariable controllers use a dynamic model in state-space representation.

Broadly, two possibilities for obtaining such a model exist: (a) black box modelling and (b) physical modelling. In physical modelling the evolution of the CSD in time is modelled using the population balance approach [4]. An accurate understanding of specific population events such as crystal birth, crystal growth and attrition, together with the crystallizer hydrodynamics is crucial in such an approach. A review of the steady state effects of these phenomena was given by Garside [5]. Unsteady state effects, however, have been generally neglected. Black box modelling offers an alternative to physical modelling. In black box modelling the process is disturbed by appropriate test signals and the resulting transients are recorded. The two are correlated using parameter estimation techniques, referred to as 'System Identification' in the field of control engineering [6] [7]. Such a procedure results in a dynamic model of the process in a state-space or equivalent representation.

A third requirement for process control is the availability of process inputs. Selective fines removal and subsequent dissolution was previously [8] identified as an important variable in CSD-manipulation. The use of size classification techniques, of which selective fines removal is just one example, appears to have an obvious need for size control. Research on size classification techniques is therefore an absolute necessity.

The purpose of this paper is to present the general concepts and techniques which are available to satisfy these three requirements. Experimental results obtained in a 20-litre crytallizer without fines removal and a 970-litre crystallizer with fines removal will be used to illustrate these concepts. In the first section the application of laser diffraction techniques for on-line size measurement will be described. In the second section, results of both the physical and black box modelling techniques will be presented. In the third and final section, an assessment of a flat-bottomed hydrocyclone for use in a size classification step is described.

\section{On-line crystal size measurement in dense slurries}

The approach chosen is to adapt an existing laser diffraction technique. This technique is selected for its ability to measure in liquids, at high speed and with reasonable reproducibility. However, high slurry densities cannot be accepted. A dilution unit has therefore been built and combined with a laser diffraction instrument, specifically a Malvern $2600 \mathrm{c}$. This sizer, whilst using a $1000 \mathrm{~mm}$ lens, has a measuring range from $0.5 \mu \mathrm{m}$ to $1900 \mu \mathrm{m}$. This range is divided into 32 size classes [9].
The combination of particle size analyzer and dilution unit determines a crystal size distribution by volume, whereas for this application absolute values of the population density of the slurry are necessary, which requires a total solids measurement. An Endress \& Hauser M-point mass flow meter was successfully used for this purpose [9].

\section{Principles of laser diffraction}

In instruments based on the laser diffraction technique, light emitted from a laser is expanded and illuminates the particle field. The particles present will either absorb or scatter the incident light according to their size, shape and refractive index. The scattered light is subsequently collimated by a Fourier transform lens and focussed onto a photodetector. In this way a composite scatter pattern developed from all the contributing particles is recorded as a function of the scattered angle. This scatter pattern must be deconvoluted in order to determine the crystal size distribution.

The success of this measuring technique depends on two factors, the accuracy of the light scattering model and the accuracy of the deconvolution technique. The light scattering model may be based on ray optics, anomalous diffraction, Fraunhofer diffraction or Mie theory. Although in principle the Mie theory is applicable for particles of any size, numerical computations may become unreliable for larger particles. During this investigation a combination of these theories, dependent mainly on the relative refractive index and particle size, was used to calculate the scatter pattern for ammonium sulfate crystals [10].

The deconvolution procedure, which translates the recorded scatter pattern into a crystal size distribution, must be both fast and reliable. In this investigation the software has been developed based on a modified matrix inversion method first described by Heuer and Leschonski [11]. The main limitation when using this technique is the smoothing of the CSD, which must be assumed. Therefore, a weighted approach has been developed. A measurement usually records and averages a large number of sweeps for each detector element. In this programme the standard deviation on the sweep values are also calculated and used to improve the accuracy of the results obtained [12].

The main limitation, currently, to these instruments, is that multiple scattering effects are ignored, which generally restricts their use to slurries of low concentration. This limitation is the main reason for development of the dilution technique.

\section{The dilution technique}

Three decisions with respect to the method of dilution were made. First, a configuration in which a sample 
is isolated in a measuring loop was chosen. This loop contains a pump which circulates the slurry and an optical cell. Second, saturated mother liquor was selected to be the dilution liquid in order to avoid dissolution or growth of crystals. This liquor is produced by feeding the stream to be measured through a hydrocyclone, passing the overflow through a set of filters which can be backflushed for cleaning and using that liquor to dilute the rest of the sample. Third, a semibatchwise dilution technique, shown in Fig. 1, was chosen [9]. Dilution is achieved by introducing clear liquid continuously to the measuring loop with a second pump. During each circulation of the slurry, the solids concentration is reduced by the ratio of the circulation flowrate and the difference between the dilution flowrate and the circulation flowrate. This mode of operation permits varying dilution ratios to be achieved by varying the opening time of valves $\mathrm{A}$ and $\mathrm{B}$. In order to avoid dissolution or growth of crystals, the whole unit is situated inside a temperature-controlled enclosure.

\section{Results}

In this papcr a sclection of the results obtained will be presented. Additional results are presented elsewhere [9]. The results of the density measurement will be first discussed. Figure 2 shows a typical example of a run of $80 \mathrm{~h}$ which included step changes in the residence time. In this case first-order responses in solids concentration are predicted [13], since the crystallizer can be considered to contain well-mixed solids. A measurement was made every $2 \mathrm{~s}$ and a first-order filter, with a time constant of $120 \mathrm{~s}$, was applied to reduce the measurement noise. From the measured slurry density the third moment of the distribution $m_{3}$, which is equivalent to the total solids concentration, is calculated by:

$m_{3}=\frac{\rho_{\text {slurry }}-\rho_{\text {lingud }}}{\left(\rho_{\text {solid }}-\rho_{\text {liquid }}\right) k_{v}}$

where $\rho_{\text {slurry }}=$ slurry density, $\rho_{\text {liquid }}=$ liquid density, $\rho_{\text {solds }}=$ solid density, $k_{\mathrm{v}}=$ shape factor.

The measured and the predicted values, given in Fig. 2, show an excellent fit. Two deviations at times of $1860 \mathrm{~min}$ and $2900 \mathrm{~min}$ do appcar which can be attributed to two process disturbances, forced batch operation and water injection respectively.

The results which were obtained using the Malvern sizer and the dilution unit illustrate a CSD-transient during crystallizer start-up, when CSD changes are most significant. The measuring frequency was 30 times per hour. From the volume distribution measured by the Malvern sizer and the total solids concentration from the density measurement, the moments of the crystal size distribution are calculated according to [13]:

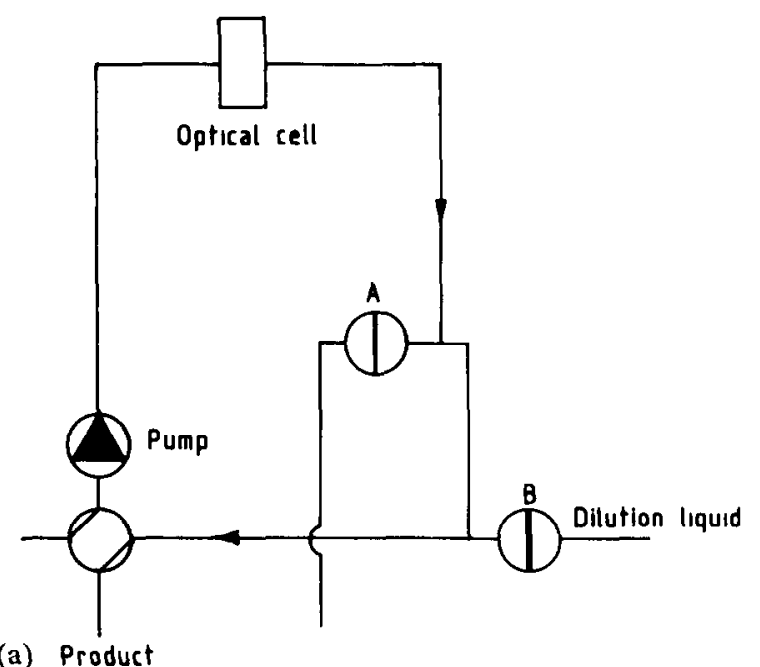

(a) Product

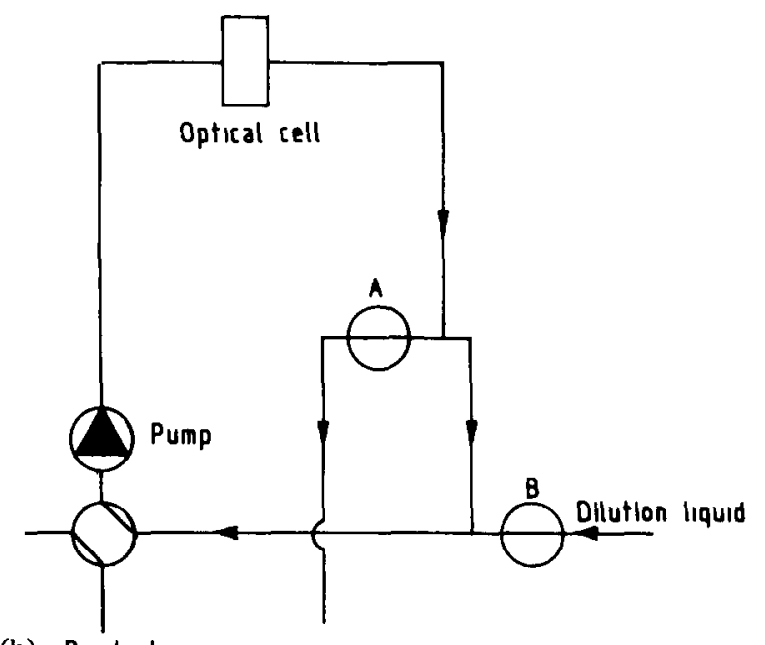

(b) Product

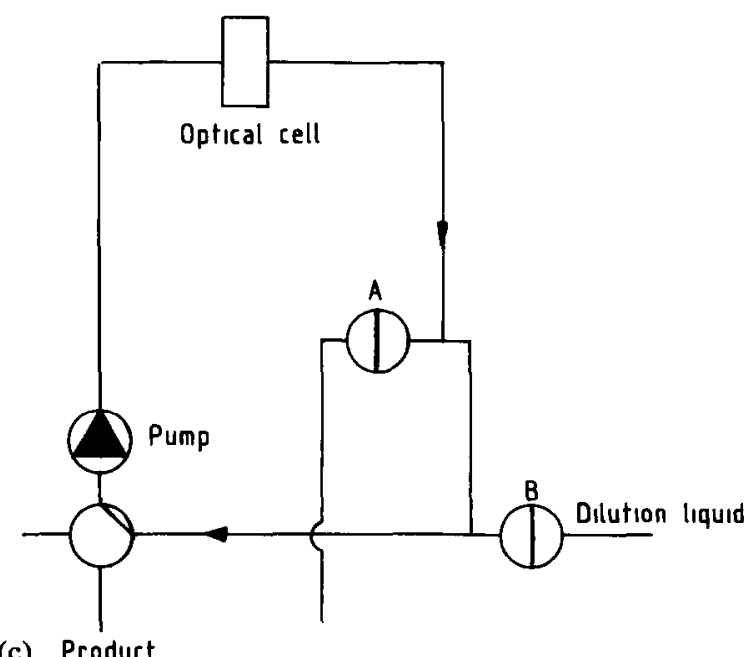

Fig. 1. Semi-batchwise dilution, (a) sampling, (b) diluting and (c) measuring. 


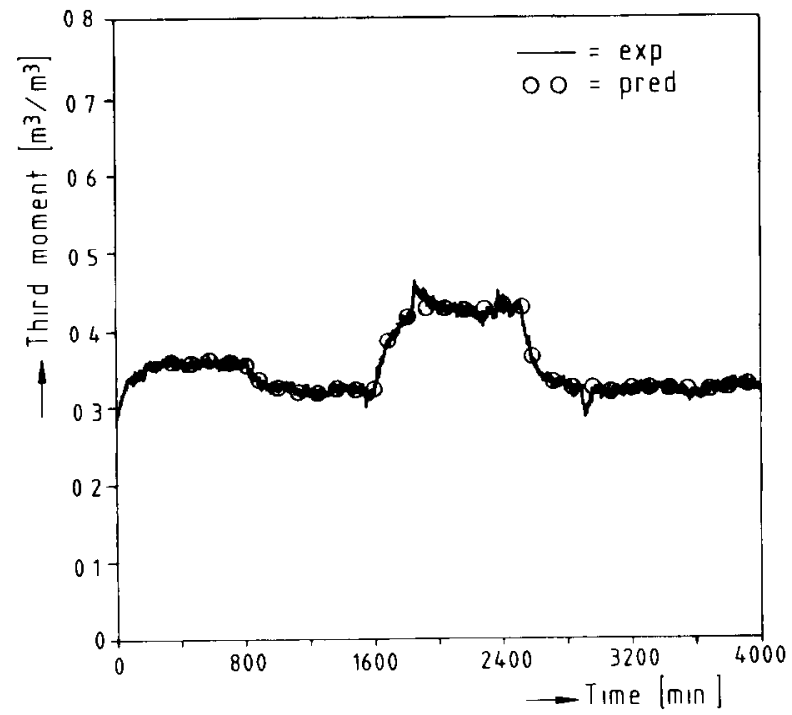

Fig. 2. Measured and predicted solids concentration using an on-line density measurement for step changes in residence tıme.

$m_{J}=m_{3} \sum_{p=1}^{32} \Delta V(p) L(p)^{-3}$

where

$\Delta V(p)=$ volume fraction of $p$ th size class

$L(p)=$ average size of $p$ th size class

In order to assess the accuracy of these results, they were compared with a sieve analysis of filtered and dried samples. These sieve results are only used to verify the trends, because the two techniques measure different particle dimensions and cannot be directly compared. Figures $3 \mathrm{a}$ and $3 \mathrm{~b}$ present the results for both techniques for the area-based average size $\left(m_{3}\right)$ $\left.m_{2}\right)$ and the mass-based average size $\left(m_{4} / m_{3}\right)$. Despite their different basis, both techniques are in good agreement and the on-line technique shows excellent reproducibility.

From the on-line, steady state values the reproducibility of the measurement can be estimated. Relative standard deviations calculated from $p$ measurements are given by:

$$
\left[\begin{array}{c}
\left.\frac{\sum_{1}^{p}\left(y_{j}(p)-\bar{y}_{j}\right)^{2}}{p-1}\right]^{1 / 2} \frac{100 \%}{\bar{y}_{J}} \\
=\text { relative standard deviation }
\end{array}\right.
$$

Values of sixth moments and the mass-based average size, $\left(m_{4} / m_{3}\right)$, are given in Table 1 .

Table 1 indicates a low relative standard deviation for the mass-based average size, the second, fourth and, of course, the third moment. This results from the fact that the laser diffraction technique mcasures
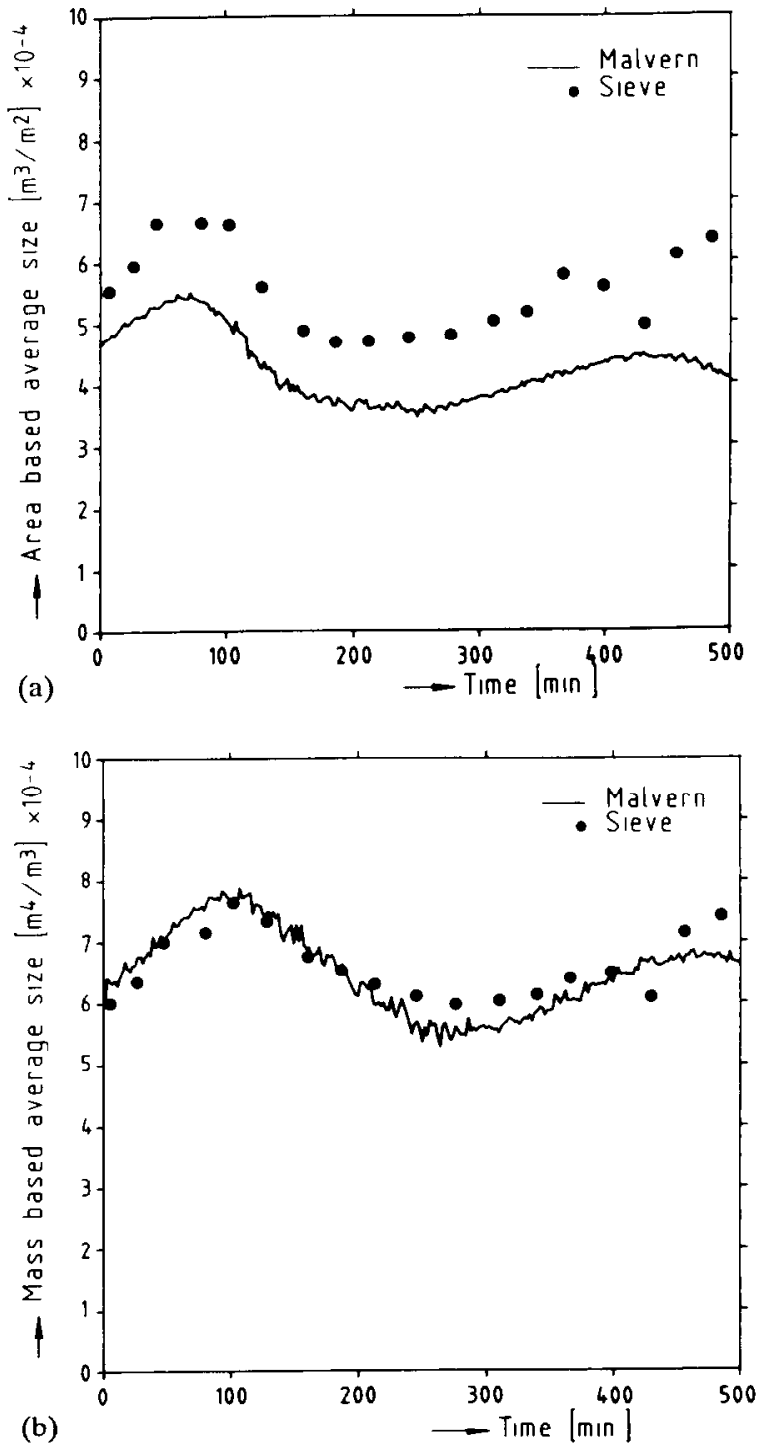

Fig. 3. The area (a) and mass (b) -based average sizes for online Malvern and off-line sieve analysis durıng crystallizer startup.

TABLE 1. Relative standard deviations of several moments calculated from the steady state portion of a crystallizer operation

\begin{tabular}{ll}
\hline Moment & $\begin{array}{l}\text { Relative standard deviation } \\
(\%)\end{array}$ \\
\hline 0 & 210 \\
1 & 4.9 \\
2 & 2.7 \\
3 & 2.3 \\
4 & 3.3 \\
5 & 6.6 \\
$4 / 3$ & 2.2 \\
\hline
\end{tabular}

a volume distribution. The zero moment is inaccurate. For this moment, the very small crystals in the first two size classes are heavily weighted. They amount to 
less than $1 \%$ of the volume distribution and inaccuracy is, therefore, very probable. The third moment has a low standard deviation because the measurement noise is contributed by the density measurement only (see eqn. (2)). In the mass-based average size, on the other hand, the contribution of the third moment, and thus the measurement noise in the third moment, cancels out.

\section{Dynamic modelling}

A second requirement for process control is an accurate dynamic model. This model can be obtained by estimating the parameters of a 'black box' model or, alternatively, by determining a model based on physical laws. Both will be illustrated in this section. First a physical model, which contains a partial differential equation to describe the population dynamics, will be presented and the unknown parameters in this model defined. Subsequently the results obtained with a nonlinear parameter estimation technique, used in the estimation of the unknown parameters, will be illustrated using results from a 20-litre crystallizer. At this scale of operation only off-line CSD-analysis was used, because of the comparatively large volume of the dilution unit.

Most multivariable control system design methods use the linear state-space model structure or a structure that is easily obtained from the state-space model. For continuous-time systems, the linear state-space model is given by a set of first-order, ordinary differential equations. In matrix notation:

$\dot{x}(t)=\mathbf{A}_{\mathrm{c}} \boldsymbol{x}(t)+\mathbf{B}_{\mathrm{c}} \boldsymbol{u}(t)$

$y(t)=\mathbf{C}_{\mathrm{c}} \boldsymbol{x}(t)+\mathbf{D}_{\mathrm{c}} u(t)$

where $\boldsymbol{x}(t)=$ state vector, dimension $(n \times 1) ; \boldsymbol{u}(t)=$ vector of process inputs, dimension $(m \times 1) ; y(t)=$ vector of process outputs, dimension $(l \times 1) ; \mathbf{A}_{\mathrm{c}}=$ state matrix, dimension $(n \times n) ; \mathbf{B}_{\mathrm{c}}=$ input matrix, dimension $(n \times m)$; $\mathbf{C}_{\mathrm{c}}=$ output matrix, dimension $(l \times n) ; \mathbf{D}_{\mathrm{c}}=$ direct transfer matrix, dimension $(l \times m) ; n=$ number of states (order of the state-space model), $m=$ number of process inputs, $l=$ number of proccss outputs. Hence, the partial differential equation describing the physical model must be transformed in order to obtain a state-space model. Several techniques are available for that purpose [14], of which two (the method of lines and the system identification techniques) will be discussed below.

For the 970-litre crystallizer, deviations, which require model refinement, do occur [15]. Although an extended model qualitatively predicts the observed deviations [15], its use for process control design is still dubious, especially since ‘black box' modelling offers an attractive alternatıve, which combınes both parameter estımation and transformation into a state-space model in one procedure. This is illustrated later.

\section{Physical modelling of the 20-litre crystallizer}

The contents of an evaporative MSMPR (Mixed Suspension-Mixed Product Removal) crystallizer which has no fines removal, are fully defined by the heat balance, the total mass balance, the solute balance and the population balance [14]. The differential equations which represent the dynamic heat, mass and solute balance can be simplified by applying two constraints for a constant volume, isothermal and Class-II crystallizer with size-independent crystal growth [13]. The Class-II assumption implies that the ammonium sulfate system is a fast-growing system in which the crystallizer concentration is constant with time and approaches equilibrium concentration. The heat and mass balance simplify into a constraint to the product flowrate:

$Q_{p}(t)=\frac{\left\{k_{1}-k_{2} C_{1}(t)\right\} Q_{t}(t)-P_{i n}(t)}{k_{3}}$

where $Q_{p}(t)=$ product flowrate, $C_{i}(t)=$ feed concentration, $Q_{i}(t)=$ feed flowrate, $P_{n n}(t)=$ heat input, $k_{1,2,3}=$ constants [13]. The solute balance reduces, making the Class-II assumption, to a growth rate constraint.

$G(t)=\frac{Q_{t}(t) C_{2}(t)-Q_{p}(t) C}{3 k_{v} V m_{2}(t)\left(\rho_{c}-C\right)}$

where $G(t)=$ crystal growth rate, $C=$ crystallizer concentration, $V=$ crystallizer volume, $m_{2}(t)=$ second moment of the CSD, $k_{v}=$ volume shape factor, $\rho_{c}=$ crystal density.

The population balance describes the CSD-dynamics. If crystal breakage and agglomeration are neglected, crystals are assumed to form at a very small size and all crystals have the same growth rate, the well-known population balance [4] results.

$\frac{\partial n(L, t)}{\partial t}+G(t) \frac{\partial n(L, t)}{\partial L}+n(L, t) \frac{Q_{p}(t)}{V}=0$

where $n(L, t)=$ population density, $L=$ crystal size.

The boundary condition for this hyperbolic partial differential equation is given by:

$n_{0}(t)=\frac{B(t)}{G(t)}$

which changes, by substitution of a power law relation for the nucleation ratc $B$ into:

$n_{0}(t)=k_{b} G^{t}(t) m_{j}^{k}(t)$

The $j$ th moment of the distribution is defined by: 
$m_{j}(t)=\int_{0}^{\infty} n(L, t) L^{\prime} d L$

Equations (6)-(11) present a dynamic model of a well-mixed crystallizer. This model contains the unknown parameters $k_{b}, i, j$ and $k$, which must be estimated from experimental results. Previously [13], a nonlinear parameter estimation technique was applied for this purpose. Using starting values for the unknown parameters, a simulation of the overall model consisting of the population balance (eqn. (8)), the nucleation model (eqn. (10)), the growth rate constraint (eqn. (7)) and the constraint for the product flowrate (eqn. (6)) can be made. The kinetic parameters are adjusted by a nonlinear optimization technique which minimizes the error between the model simulation and the measured responses until some stop criterion is attained. A suitable measure of the deviation of the data from the model (objective function) must be defined. In this investigation a least squares objcctive function, which is the sum of squares of the residuals, was chosen. Typically, simulated datapoints are subtracted from the experimental ones, squared and summed. Weighting factors can be used to emphasize reliable observations and to scale the data. An identical criterion, denoted 'output error', was used in the black box modelling approach discussed in the next section.

$\operatorname{Sum}=\sum_{p=1}^{n} \sum_{j=1}^{z} w_{j}\left[y_{j}\left(t_{p}\right)-\hat{y}_{j}\left(t_{p}\right)\right]^{2}$

where $n=$ number of datapoints, $y=$ experimental value, $\hat{y}=$ predicted value, $w_{l}=$ weighting factor for $j$ th signal, $z=$ number of signals.

The mass-based average size was chosen to be fitted. The third moment is used in the calculation of the nucleation rate, as indicated in eqn. (13). More details of the optimization procedure have been presented elsewhere [13]. Figure 4 presents the fit for an experiment in which the heat input was increased stepwise from $2.0 \mathrm{~kW}$ to $3.3 \mathrm{~kW}$ at an operating time of $16500 \mathrm{~s}$. The nucleation model can be fitted by the following expression:

$n_{0}=2.710^{25} \mathrm{G}^{1.5} m_{3}^{20}$

Figure 4 presents an excellent fit for the mass-based average size and thus, an acceptable representation of this parameter was achieved. However, the model equations (6)-(11) did not have the required state-space representation (eqns. (4), (5)). Several techniques are available for the transformation of the partial differential equation [14]. If a fines removal procedure must be accounted for and general applicability is required, two major techniques remain, the method of lines and system

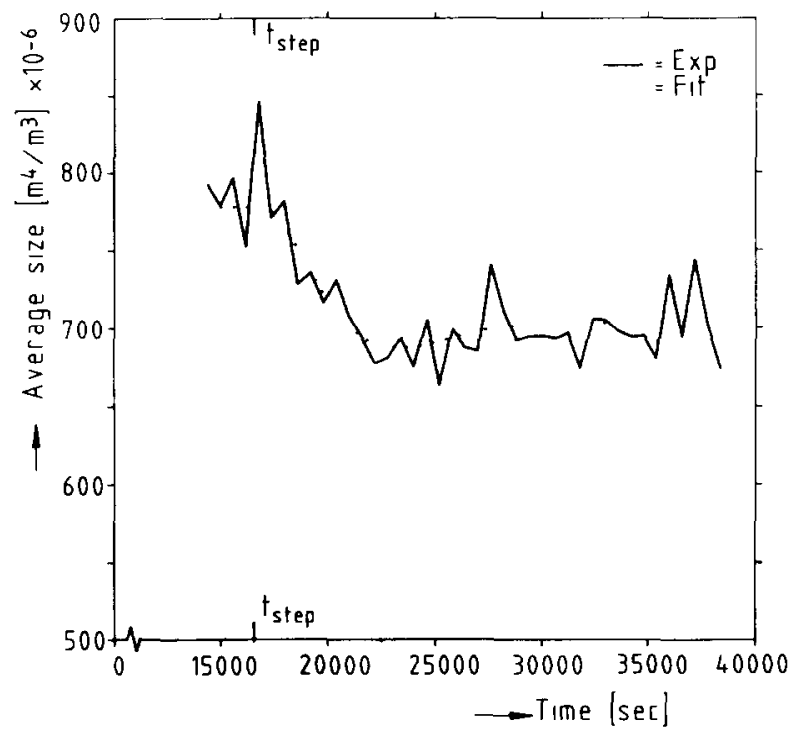

Fig. 4. The experimental vs. fitted mass-based average size for an optimized parameter combination according to eqn. (13) for a step in heat input from $2.0 \mathrm{~kW}$ to $3.3 \mathrm{~kW}$ at $t=16500$ seconds.

identification techniques [14]. In the method of lines, the partial derivative with respect to size, eqn. (8), is replaced by a finite difference approximation in discrete gridpoints $\left(L_{p}\right)$. A first-order, backward difference, for example, is given by:

$\frac{\partial n\left(L_{p}, t\right)}{\partial L}=\frac{n\left(L_{p}\right)-n\left(L_{p-1}\right)}{L_{p}-L_{p-1}}$

By substituting this approximation into eqn. (8), a number of ordinary differential equations for the population dynamics in the gridpoints result which approximate the original partial differential equation. This set of equations must be linearized at an operating point in order to obtain the required state-space representation [14]. The main disadvantage of this approach is the large number of equations required to approximate eqn. (8) with sufficient accuracy, in other words the high order of the state-space model obtained. Such high orders can cause severe problems in the design and implementation of a multivariable controller.

System identification techniques offer an attractive alternative to the method of lines, in that the final state-space model is of much lower order [14]. As already stated, these techniques can also be applied directly to the experimental data, which constitutes a more direct approach. This approach will now be illustrated using experimental data obtained using the 970-litre crystallizer.

\section{Black box modelling of the 970-litre crystallizer}

System identification is a widely used modelling technique in control engineering $[6,7]$. In system identification, the response of the outputs of a system to the 
input signals are approximated by a black box model. The parameters in this model are determined by minimizing a criterion function which is based on some difference between the measured input-output data and the responses predicted by the model. Different model structures can be chosen and, dependent on this structure, different criteria can be employed. In this investigation, a three-step identification procedure was used to obtain the state-space model. The system identification procedures are based on discrete-time models and thereforc thc parameters in the discretetime equivalent of eqns. (4) and (5) are estimated. This model is given by:

$\boldsymbol{x}\{(k+1) \Delta T\}=\mathbf{A}_{d} \boldsymbol{x}(k \Delta T)+\mathbf{B}_{d} \boldsymbol{u}(k \Delta T)$

$\boldsymbol{y}(k \Delta T)=\mathbf{C}_{d} \boldsymbol{x}(k \Delta T)+\mathbf{D}_{d} u(k \Delta T)$

where $\Delta T=$ the sample interval.

In the first step, an ARX-model is used, which has the form [6]:

$H\left(z^{-1}\right) \boldsymbol{y}(k \Delta T)=F\left(z^{-1}\right) \boldsymbol{u}(k \Delta T)$

where $z^{-v}$ is defined by $z^{-v} \boldsymbol{y}(k \Delta T)=\boldsymbol{y}\{(k-v) \Delta T\}$ and

$H\left(z^{-1}\right)=\mathrm{I}_{l}+H_{1} z^{-1}+H_{2} z^{-2}+\ldots H_{q} z^{-q}$

$F\left(z^{-1}\right)=F_{0}+F_{1} z^{-1}+F_{2} z^{-2}+\ldots F_{p} z^{-p}$

$\mathbf{I}_{l}=$ the $(l \times l)$ identity matrix.

The parameters in $H\left(z^{-1}\right)$ and $F\left(z^{-1}\right)$ are estimated by minimizing the equation error criterion [6]. In the second step, this model is transformed into a state-space model by making an approximate realization [16]. This step includes the determination of the order of the state-space model (the dimension of the matrix $\mathbf{A}_{d}$ ), which is determined by inspection of the singular values of the Hankel matrix which is the matrix containing the impulse responses of the ARX-model. In the third step, the state-space model obtained from the second step is used as an initial parameter combination to fit the discrete-time state-space model to the actual data. Here the output error criterion [6] is minimized (See eqn. (12)). Because the output error criterion is highly nonlinear in the parameters of $\mathbf{A}_{d}$, the first two steps were required to determine the order of the state-space model and to obtain a good initial parameter set for this third step, only.

During the collection of experimental data to be used for system identification, Pseudo-Random Binary Noise (PRBN) signals are commonly used, as illustrated in Fig. 5. These signals are added to the steady state values of the input signals. PRBN signals are widely used because they are easy to generate and have characteristics which approximate those of uncorrelated white noise signals. The advantage of using uncorrelated input signals is that they can be added to the process

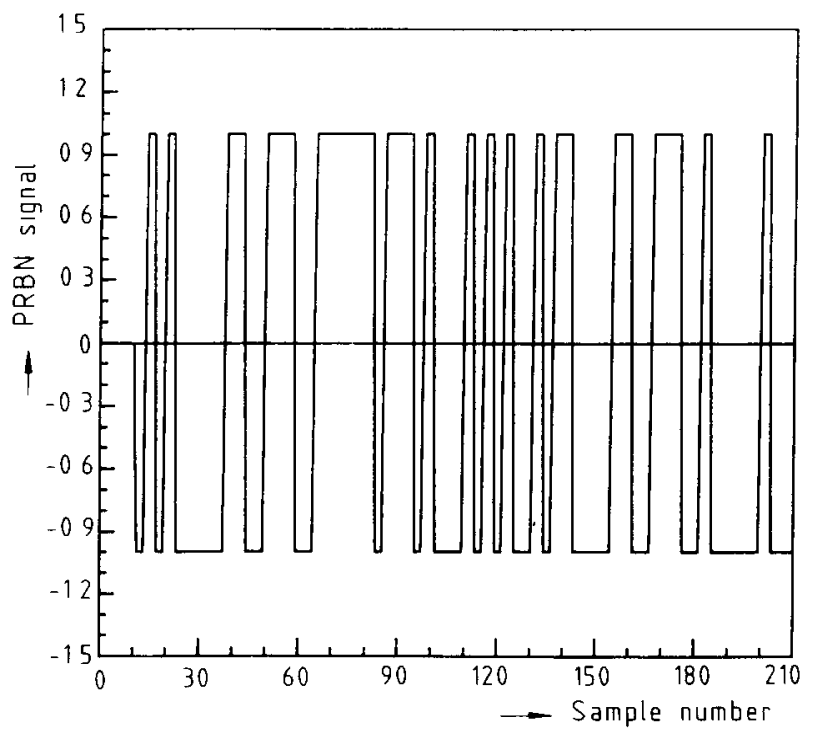

Fig. 5. Example of a PRBN signal.

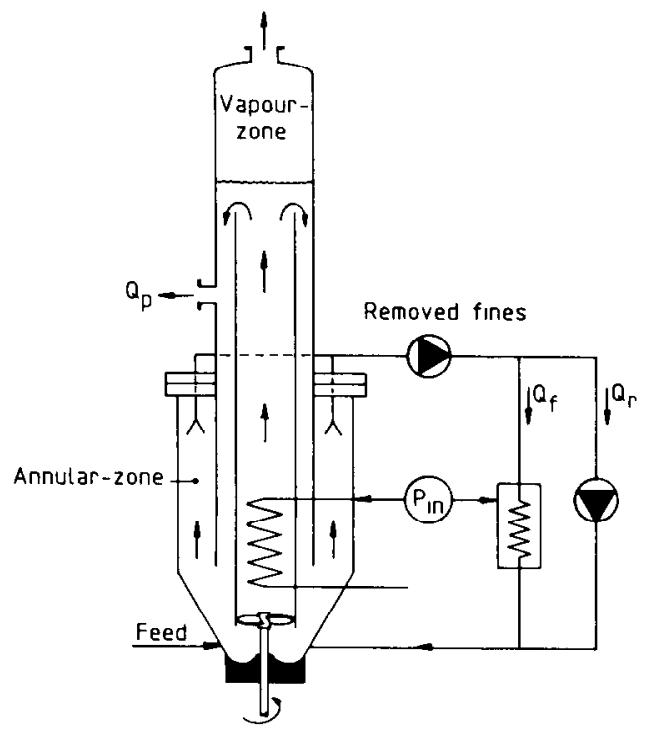

Fig. 6. The 970-litre crystallizer.

inputs simultaneously, without affecting the ability of the identification algorithm to distinguish the contributions of each individual input to the output signals. This allows an efficient use of data.

Three inputs are available for CSD-control in the present investigation, the heat input to the process $\left(P_{\mathrm{in}}\right)$, the product flowrate $\left(Q_{\mathrm{p}}\right)$ and the rate of fines dissolution $\left(Q_{f}\right)$. They are illustrated in Fig. 6. Available software, described in [17] and [18], was used in the three step identification procedure. Figure 7 shows the measured third moment and the prediction by the identified state-space model, whereas Fig. 8 depicts the results for the mass-based average size. Both figures demonstrate a good fit to the data, which illustrates the usefulness of the technique in estimating a dynamic 


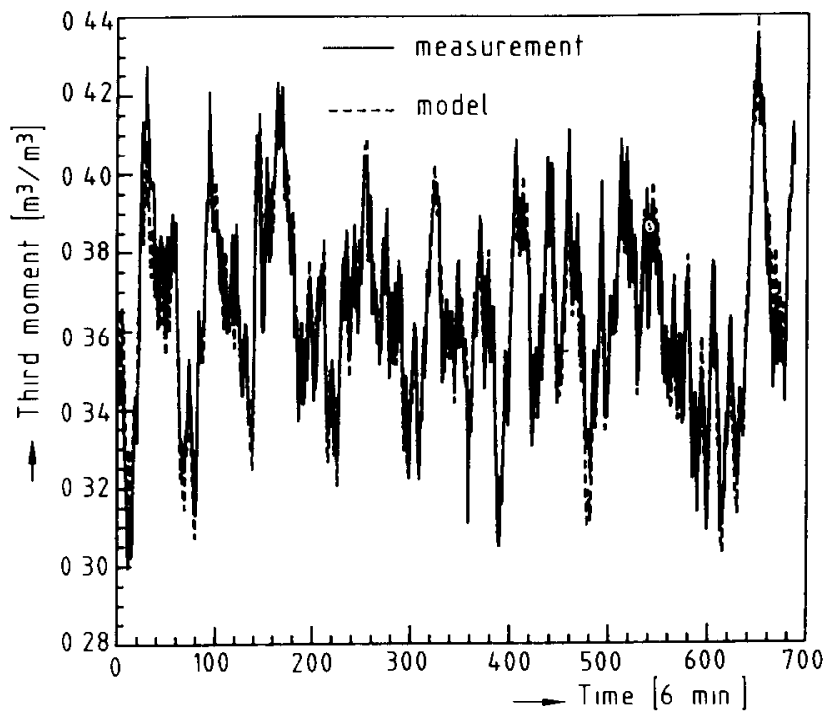

Fig. 7. Measured responses and identification results for the third moment of the size distribution.

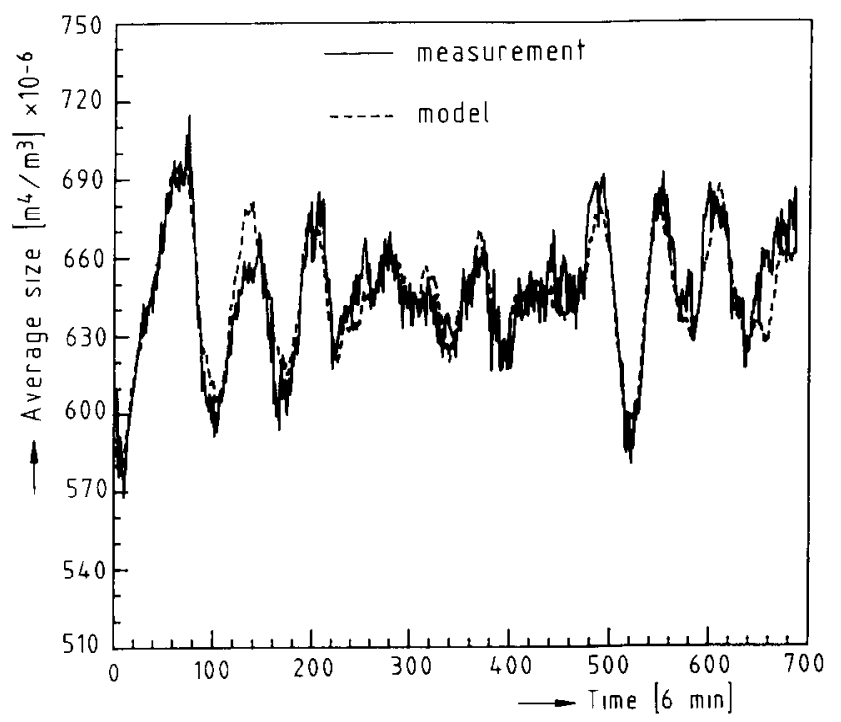

Fig. 8. Measured responses and identification results for the mass-based average size.

model of a complicated crystallization process. Additional results are presented elsewhere [19].

\section{Hydrocyclone separation}

The number of inputs which are available for controlling crystallization processes is limited. The crystallizer temperature, residence time and rate of evaporation affect the crystal size distribution (CSD) through overall changes in the nucleation rate and growth rate, and have therefore a confined effect. Size classification techniques, that is the selective removal and dissolution of fines, or product classification do discriminate with respect to size. However, the effect of fines removal and dissolution is also limited. The experimental results shown in Fig. 8 illustrate the relatively small variation in an average size of $650 \mu \mathrm{m}$ which can be attained with variations in the input signals. This indicates that current inputs are insufficient to achieve full control. There is a clear need for a product classification step whereby coarse crystals are removed at a defined size.

Hydrocyclones have a small volume, are simple to operate and are standard size-classification equipment in applications such as closed-circuit grinding. Recent development of a flat-bottomed hydrocyclone [20], which permits classification in a coarse size range, suggests the possibility of its use to control Crystal Size Distribution. Furthermore, throttling a flat-bottom hydrocyclone does not necessarily provoke blockage but allows continuous control of the cut size. There is a clear incentive for its use in this application, since it may provide an additional process input.

We now present a selection of experimental results previously reported [21], obtained when using a $75 \mathrm{~mm}$, flat-bottom cyclone (RWB 1613) provided by the Amberger Kaolin Werke (AKW). The cyclone was tested in a batch opcration, using the product formed by the 20-litre crystallizer as feed. The purpose of these results is to illustrate the ability of the hydrocyclone to separate a coarse cut size and to illustrate the effect of a change in underflow diameter on its separation characteristics.

The performance of a hydrocyclone is generally characterized by means of a grade-efficiency, or Tromp, curve which is the fractional mass recovery expressed as a function of particle size. By definition:

$\theta=\frac{\text { solids underflow }}{\text { solids feedflow }}$

The Tromp curve for the removal of coarse particles with the underflow is defined by:

$$
\frac{\theta \Delta V(p)_{u}}{\theta \Delta V(p)_{u}+(1-\theta) \Delta V(p)_{o}}=\text { efficiency for interval } p
$$

where $\Delta V(p)_{o}=$ volume fraction in the $p$ th interval reporting to the overflow; $\Delta V(p)_{u}=$ volume fraction in the $p$ th interval reporting to the underflow.

Figure 9 shows the grade efficiency curves determined at a flowrate of $1.41 \mathrm{~s} \mathrm{~s}^{-1}$ using apex diameters of $10 \mathrm{~mm}$ and $16 \mathrm{~mm}$ respectively at a cyclone length of $0.25 \mathrm{~m}$. The results illustrate the variation which can be obtained by varying the apex diameter. The effect of throttling is significant, which suggests that an additional process input can indeed be created. A shift in the grade-efficiency curve of about $100 \mu \mathrm{m}$ is found to be possible. Figure 10 shows the size distributions of the feed, the underflow and the overflow, 


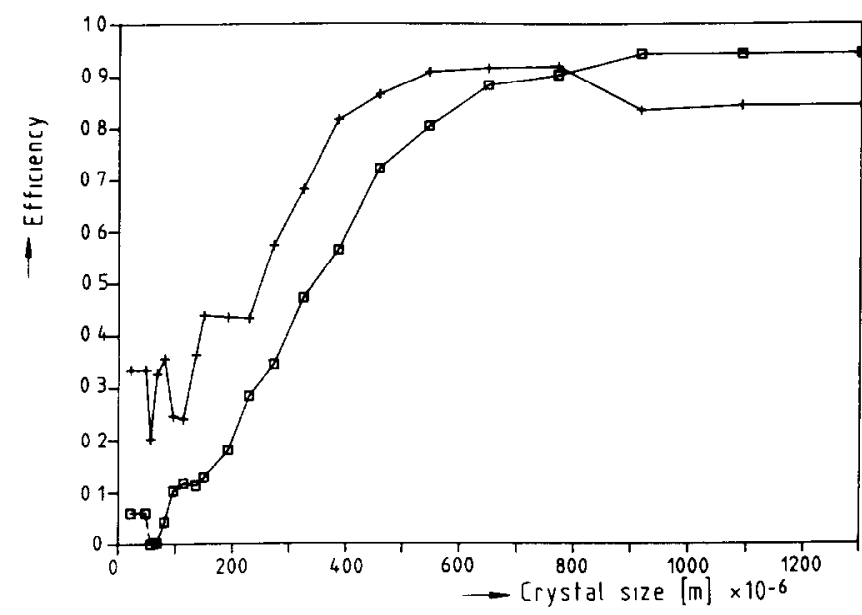

Fig. 9. Two grade-efficiency curves for product classification at an apex diameter of $(\square) 10 \mathrm{~mm}$ and $(+) 16 \mathrm{~mm}$ at a flowrate of $1.4 \mathrm{l} \mathrm{s}^{-1}$

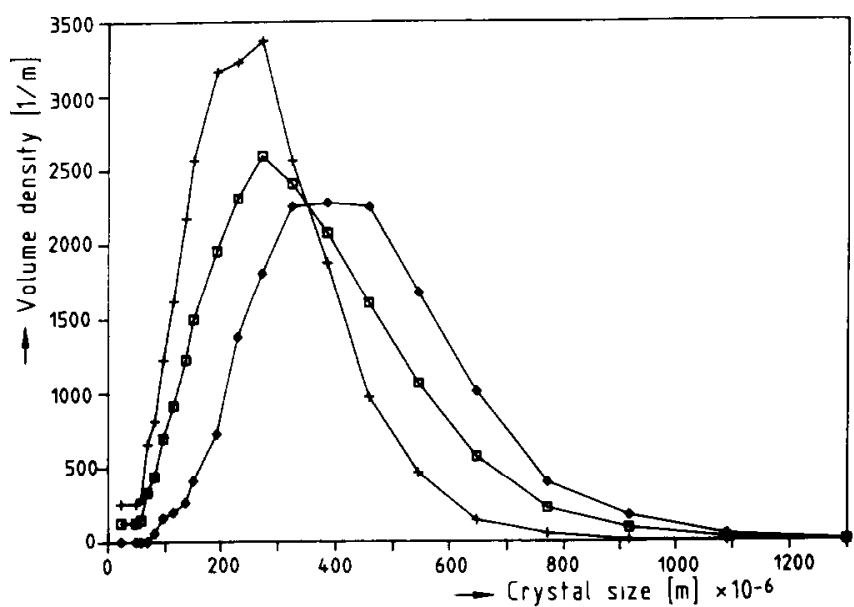

Fig. 10. The volume distributions in the $(\square)$ feed, $(+)$ overflow, and $(\diamond)$ underflow, at a flowrate of $1.41 \mathrm{~s}^{-1}$ and an apex diameter of $10 \mathrm{~mm}$.

using an apex diameter of $10 \mathrm{~mm}$ and a flowrate of $1.4 \mathrm{~s}^{-1}$. At these conditions the difference in size between the feed and the underflow is most pronounced at a value of $100 \mu \mathrm{m}$.

These experimental results demonstrate the ability of a flat-bottom hydrocyclone to separate the coarse fraction of ammonium sulfate crystals from a slurry which contains crystals of a wide size range. It appears that the grade-efficiency curve, which predicts the probability of a particle reporting to the underflow of the cyclone, can be adjusted by a change in the underflow diameter of the hydrocyclone. These two observations lead to the suggestion of using hydrocyclone separation to reduce the crystal size distribution which is produced in crystallizers, that is, using a variable underflow diameter as an additional input for process control. This is a current thrust of these investigations.

\section{Conclusions}

The application of an on-line CSD-measuring technique, combining a laser diffraction instrument, a specific scatter and deconvolution model, and a dilution unit, has been illustrated.

Two complementary modelling techniques have been presented. Physical modelling requires a detailed model of the process. If such a model is available, it has the advantage of being a more transparent technique. It delivers, however, a model which must still be transformed. Black box modelling, on the other hand, does not require a detailed physical model and can be applied directly to experimental data to estimate a model in the required state-space representation. As such, the technique has a wider range of applications.

The effect of existing inputs such as the product flowrate, the heat input and the rate of fines dissolving proved to be limited. The use of hydrocyclone separation to improve CSD-control is therefore now being developed.

\section{Acknowledgements}

The authors wish to thank the Netherlands Technology Foundation (STW), Akzo, Dow, DSM, Dupont, Rhône Poulenc, Suiker Unie and Unilever for their financial support of the UNIAK research programme.

\section{List of symbols}

\begin{tabular}{|c|c|}
\hline $\mathbf{A}_{c}, \mathbf{A}_{\mathrm{d}}$ & state matrices dimension $(n \times n)$ \\
\hline $\mathbf{B}_{\mathrm{c}}, \mathbf{B}_{\mathrm{d}}$ & input matrices dimension $(n \times m)$ \\
\hline$B$ & nucleation rate $\left[\# /\left(\mathrm{m}^{3} \mathrm{~s}\right)\right]$ \\
\hline $\mathbf{C}_{c}, \mathbf{C}_{\mathrm{d}}$ & output matrices dimension $(l \times n)$ \\
\hline & direct transfer matrices dimension $(l \times m)$ \\
\hline$G$ & $\begin{array}{l}\text { polynomial matrix dimension }(l \times m) \\
\text { growth rate }\left[\mathrm{m} \mathrm{s}^{-1}\right]\end{array}$ \\
\hline$H$ & polynomial matrix dimension $(l \times m)$ \\
\hline$i, k$ & empirical constants \\
\hline$j$ & used to denote the moments \\
\hline$k_{b}$ & nuclcation constant \\
\hline$k_{v}$ & shape factor $(=0.26)$ \\
\hline & index \\
\hline$k_{1}, k_{2}, k_{3}$ & constants \\
\hline$l$ & number of process outputs \\
\hline$L$ & crystal size $(\mathrm{m})$ \\
\hline Msl & slurry density ( $\mathrm{kg} \mathrm{m}^{-3}$ slurry) \\
\hline$m$ & number of process inputs \\
\hline$m_{j}$ & $j$ th moment of the distribution $\left(m^{\prime} \mathrm{m}^{-3}\right)$ \\
\hline$n$ & $\begin{array}{l}\text { number of states (order of the state space } \\
\text { model) }\end{array}$ \\
\hline$n_{0}$ & population density at zero size $\left(\# / \mathrm{m}^{4}\right)$ \\
\hline
\end{tabular}


$n(L, t) \quad$ population density at size $L\left(\# / \mathrm{m}^{4}\right)$

$P_{\mathrm{nn}} \quad$ heat input (Watt)

$p \quad$ index

$q \quad$ index

$Q \quad$ flowrate $\left(\mathrm{m}^{3} \mathrm{~s}^{-1}\right)$

$t \quad$ time (s)

$V \quad$ crystallizer volume $\left(\mathrm{m}^{3}\right)$

$u(t) \quad$ vector of process inputs, dimension $(m \times 1)$

$w_{j} \quad$ weighting factor

$\boldsymbol{x}(t) \quad$ state vector, dimension $(n \times 1)$

$y(t) \quad$ vector of process outputs, dimension $(l \times 1)$

$z$

\section{Greek letters}

$\Delta V\left(L_{\rho}, t\right) \quad$ volume fraction in the $p$ th size class

$\Delta L_{p} \quad$ width of a size interval $p(\mathrm{~m})$

$\Delta T \quad$ sampling interval (s)

$\rho$ density $\left(\mathrm{kg} \mathrm{m}^{-3}\right)$

$\rho_{\mathrm{c}} \quad$ crystal density $\left(\mathrm{kg} \mathrm{m}^{-3}\right)$

$\tau \quad$ residence time $(\mathrm{s})$

$\theta \quad$ mass recovery

$\begin{array}{ll}\text { Indices } & \\ \mathrm{i} & \text { denotes feed } \\ \mathrm{o} & \text { denotes overflow } \\ \mathrm{p} & \text { denotes product } \\ \mathrm{u} & \text { denotes underflow }\end{array}$

no index denotes crystallizer contents

\section{References}

1 E. J. De Jong, Proc. World Congress III Chem. Eng., Tokyo, 1986, The Society of Chemical Engineers, Japan, 1986, Vol. 2, pp. $1080-1083$.

2 A. D. Randolph, L. Chen and A. Tavana, AIChE J., 33 (1987) 583.

3 S. Rohanı and K. K. Lee, in G. L. Strathdee, M O. Kleın and L A. Melis (eds.), Crystallization and Precipttation, Pergamon, Oxford, 1987 , p. 11-20.

4 A. D. Randolph and M A Larson, Theory of Particulate Processes, Plenum, New York, 1971

5 J. Garside, Chem. Eng. Sct, 40 (1985) 3

6 L. Ljung, System Identification Theory for the User, PrenticeHall, New York, 1987.

7 T. Soderstrom and P. Stoıca, System Identification, Prentice. Hall, New York, 1989.

8 B. G. M. De Leer, Ph.D. Thess, Delft Unıversity of Technology, 1981.

9 J. Jager, S. de Wolf, H. J. M. Kramer and E. J. de Jong, Powder Technol., 62 (1990) 155.

10 A. Boxman and B. Scarlett, in O. D. D. Soares (ed.), Proc Laser Technologies in Industry, Society of Photo-Optical Instrument Engineers, Vol. 952, June 1988, pp. 520-523.

11 M Heuer and K. Leschonski, Part. Charact, 2 (1985) 7.

12 A. Boxman, H G. Merkus, P. J T. Verherjen and B Scarlett, in Proc. Partec 4 European Symposium Particle Characterization, Numberg, 1989, pp. 549-560.

13 J. Jager, S. de Wolf, H. J. M. Kramer and E. J. de Jong, Chem. Eng. Sct. 46 (1991) 817.

14 S. de Wolf, J. Jager, H. J. M. Kramer, R. Eek and O. H. Bosgra, in Proc. IFAC Symp Dynamics and Control of Chemical Reactors, Dustllation Columns and Batch Processes, 21-23 August 1989, Maastricht.

15 J. Jager, S. de Wolf, H. J. M. Kramer and E. J. de Jong, AIChE J., 37 (1991) 182

16 A. A. H. Damen and A. K. Hajdasinski, in Proc. 6th IFAC Symp. Identfication and System Parameter Estimation, Wash. ington DC, 1982, pp. 903-908.

17 P. J. M. van den Hof, DUMSI-package for off-line multvanable system tdentification. Delft University of Technology, Laboratory for Measurement and Control, Delft, 1989.

18 H. Aling, RPEPAC User's manual, Delft University of Technology, Laboratory for Measurement and Control, Report N-304, Delft, 1989.

19 S. de Wolf, J. Jager, O. H. Bosgra and H. J. M. Kramer, in Proc. 11th Int. Symp. Crystallization, Garmisch-Partenkirchen, September, 1990.

20 H. F. Trawinsky, Fultr. Sep., Jan/Feb (1985).

21 J. Jager, S. de Wolf, H. J. M. Kramer and E. J. de Jong, in A. S. Myerson and K. Toyohara (eds), Crystallization as a Separations Process, American Chemical Soc., Washington D.C., 1990 , pp. 130-143. 